\title{
Changes in Tonicity of Perfusion Medium Cause Prolonged Opening of Calcium Channels of the Rat Chromaffin Cells to Evoke Explosive Secretion of Catecholamines
}

\author{
Arun R. Wakade, R. K. Malhotra, T. R. Sharma, and Taruna D. Wakade \\ Department of Pharmacology, State University of New York, Downstate Medical Center, Brooklyn, New York \\ 11203
}

Secretion of catecholamines (CA) from the isolated rat adrenal gland during and after perfusion with hypertonic Krebs bicarbonate solution was studied. Perfusion with hypertonic solution made by adding either $156 \mathrm{~mm}$ sodium chloride, choline chloride, arginine hydrochloride, sodium thiocyanate, or $312 \mathrm{~mm}$ sucrose had no effect on the spontaneous secretion of $\mathrm{CA}$. The secretion evoked by splanchnic nerve stimulation $(10 \mathrm{~Hz}$ for 30 sec) and nicotine ( $2 \mu \mathrm{g})$ remained unaffected during perfusion with hypertonic Krebs solution (156 $\mathrm{mm}$ excess $\mathrm{NaCl})$. After perfusion of the adrenal gland with hypertonic Krebs solution for $1 \mathrm{hr}$, if the medium was switched to normal Krebs solution the secretion of $\mathrm{CA}$ increased from about 15 to $450 \mathrm{ng}$; it remained elevated for over $1 \mathrm{hr}$ and eventually returned to the control level after $150 \mathrm{~min}$. Secretion of CA obtained in normal Krebs solution after perfusion of the adrenal gland with hypertonic medium was not affected by atropine plus hexamethonium, splanchnectomy, or tetrodotoxin.

After perfusion of the adrenal gland with Ca-free hypertonic ( $275 \mathrm{~mm} \mathrm{NaCl}$ ) Krebs solution for $1 \mathrm{hr}$, if the medium was changed to normal Krebs solution the secretion of $\mathrm{CA}$ increased from 10 to about $4000 \mathrm{ng}$ in the first $10 \mathrm{~min}$. The enhanced secretion was associated with an almost $70 \%$ reduction in the CA content of the adrenal medulla. A massive secretion of $\mathbf{C A}$ obtained after switchover from hypertonic to normotonic solution was not associated with an increase in lactate dehydrogenase content in the perfusate, nor was there any reduction in the lactate dehydrogenase content of the adrenal medulla. Immediately after the switchover from hypertonic to normotonic medium, the accumulation of $\mathrm{Ca}^{45}$ increased 6-fold over the control accumulation. The increase in $\mathrm{Ca}^{45}$ accumulation was detected as late as 120 min after the switchover, and was accompanied by an increase in the secretion of $\mathrm{CA}$. The most likely explanation for these findings is that Ca channels, once opened after the switchover, inactivate very slowly and allow $\mathrm{Ca}$ ions to flow inside the chromaffin cells. The accumulation of tetraphenylphosphonium (C ${ }^{14}$-TPP), a marker for the membrane potential, was $86 \mathrm{pg} / \mathrm{mg}$ in the adrenal medulla perfused with normal Krebs solution, and decreased to $32 \mathrm{pg} / \mathrm{mg}$ in the presence of $55 \mathrm{~mm} \mathrm{~K}$-Krebs solution. After perfusion with hypertonic medium, the accumulation of C 14 -TPP increased to about 190 $\mathrm{pg} / \mathrm{mg}$. Reperfusion with normal Krebs solution decreased the accumulation to about $105 \mathrm{pg} / \mathrm{mg}$. The increase in $\mathrm{C}^{14}-\mathrm{TPP}$ accumulation observed in hypertonic medium was prevented by inclusion of $30 \mathrm{~mm} K$ in the medium. High-K treatment also

\footnotetext{
Received Oct 15, 1985; revised Feb. 14, 1986; accepted Feb. 24, 1986

This article is dedicated to the memory of the late Professor S. M. Kirpekar.

Correspondence should be addressed to Arun R. Wakade, Department of Pharmacology, State University of New York, Downstate Medical Center, 450 Clarkson Avenue, Brooklyn, NY 11203.

Copyright (C) 1986 Society for Neuroscience 0270-6474/86/092625-10\$02.00/0
}

prevented the explosive secretion of CA obtained after switchover from hypertonic to normotonic medium. It is suggested that perfusion of the adrenal medulla with hypertonic solution causes hyperpolarization of the chromaffin cell membrane, and switchover to normotonic medium moves the membrane potential toward the original resting level. This relative, rather than absolute, change in the membrane potential is of sufficient magnitude to open Ca channels and to raise intracellular $\mathrm{Ca}$ concentration high enough to evoke massive secretion of $\mathrm{CA}$.

A number of investigators have used perfused adrenal glands of different species to evoke secretion of catecholamines (CA) by a variety of maneuvers in order to study the role of $\mathrm{Ca}$ ions in the secretory process (Baker and Rink, 1975; Douglas and Rubin, 1963; Kidokoro et al., 1979; Kirpekar and Cervoni, 1963; Marley and Paton, 1961; Robinson, 1967; Wakade, 1981a; Wilson and Kirshner, 1977). In the isolated perfused adrenal gland of the rat, it has been possible to evoke secretion by (1) activation of nicotinic and muscarinic receptors by nicotine, muscarine, or by ACh liberated from presynaptic splanchnic nerve terminals (Wakade and Wakade, 1983); (2) increasing the extracellular concentration of $K$, which is believed to cause depolarization of the chromaffin cell membrane (Kidokoro et al., 1979; Wakade and Wakade, 1983); (3) direct electrical stimulation of chromaffin cells using pulses of high strength or prolonged duration, which probably lead to nonpropagated local depolarization of the chromaffin cell membrane (Wakade and Wakade, 1982a); and (4) changing the ionic environment of the chromaffin cells by blocking the activity of the Na-K pump by means of cardiac glycosides or K-deprivation (Wakade, 1981b). In all of the above procedures, which have led to the secretion of CA, the indispensable role of calcium has been established.

The present study describes an entirely different approach to evoking secretion of $\mathrm{CA}$ in a Ca-dependent manner. We show that a switchover from hypertonic to normotonic perfusion medium results in a massive amount of CA secretion. A series of experiments has been designed to elucidate the mechanism of this secretion of CA. Preliminary reports of some of these results have already been presented (Sharma and Wakade, 1984; Wakade et al., 1985).

\section{Materials and Methods}

\section{Retrograde perfusion of the adrenal gland}

The left adrenal gland of the male rat $(300-400 \mathrm{gm})$ was perfused retrogradely, as described earlier (Wakade, 1981a). Briefly, rats were anesthetized with ether, the left renal vein was cannulated, and the tip of the cannula remained near the junction of the renal and adrenal veins. All other blood vessels were ligated. The adrenal gland, along with tied blood vessels and the cannula, was removed from the rat and placed on a metal plate mounted in a Lucite chamber. The metal plate was made up of $\mathrm{Ag} / \mathrm{AgCl}$ and served as one of the electrodes for stimulation 
of the gland; another plate electrode was placed on top of the gland (see below). The chamber was maintained at $37^{\circ} \mathrm{C}$ by circulating heated water. The gland was perfused at $0.35 \mathrm{ml} / \mathrm{min}$ by means of a Sigma motor pump. Perfusate cscaped from a slit made in the adrenal cortcx and was collected in chilled tubes.

\section{Perfusion media}

Krebs bicarbonate solution was used for the perfusion of the adrenal glands. The composition of the solution was as follows (mm): $\mathrm{NaCl}$, $119 ; \mathrm{KCl}, 4.7 ; \mathrm{CaCl}_{2}, 2.5 ; \mathrm{MgSO}_{4}, 1.2 ; \mathrm{KH}_{2} \mathrm{PO}_{4}, 1.2 ;$ glucose, $11 ; \mathrm{NaHCO}_{3}$, 25. Hypertonic solution was made by adding $156 \mathrm{~mm}$ of either sodium chloride, choline chloride, sodium thiocyanate, or arginine hydrochloride to Krebs bicarbonate solution. Hypertonic solution was also made by adding 156 and $312 \mathrm{~mm}$ sucrose. Ca-free medium was prepared by omitting $\mathrm{CaCl}_{2}(2.5 \mathrm{~mm})$ and adding $1 \mathrm{~mm}$ EGTA to either normal or hypertonic Krebs solution. $K$ concentration of the medium was increased to 30 or $55 \mathrm{~mm}$ by adding excess amounts of $\mathrm{KCl}$ and omitting equivalent amounts of $\mathrm{NaCl}$, unless stated otherwise. All solutions were bubbled with $95 \% \mathrm{O}_{2}+5 \% \mathrm{CO}_{2}$, and the final $\mathrm{pH}$ was $7.4 \pm 0.1(n=$ 13). In all cases the adrenal gland was perfused for $30 \mathrm{~min}$ with Krebs solution before the beginning of the experiment.

\section{Stimulation of the adrenal gland}

Stimulation of the adrenal gland was achieved by connecting the plate electrodes to a Grass Model S88 stimulator. Stimulation parameters were 300 shocks at different frequencies $(1.0 \mathrm{msec}$ duration and 180 $\mathrm{mA}$ strength). In other cases, $2 \mu \mathrm{g}$ nicotine was injected into the perfusion stream to evoke CA secretion. Perfusates were collected for varying periods of time, as described below.

\section{$C^{14}-T P P$ accumulation}

Thirty minutes after perfusion with Krebs solution, the medium was switched over to Krebs solution containing $2.2 \mu \mathrm{g} / \mathrm{ml}$ tetraphenylphosphonium (C ${ }^{14}-\mathrm{TPP}$ ) (specific activity, $19.2 \mathrm{mCi} / \mathrm{mmol}$; New England Nuclear) for $45 \mathrm{~min}$, and then the gland was perfused with $\mathrm{Ca}$-free Krebs solution at $4^{\circ} \mathrm{C}$ for $10 \mathrm{~min}$. The adrenal medulla was separated, weighed, and homogenized in $0.1 \mathrm{~N}$ perchloric acid $(1 \mathrm{ml})$, and after centrifugation $(2000 \mathrm{rpm})$, the supernatant $(0.5 \mathrm{ml})$ was counted in a Beckman liquid scintillation counter (Beckman Model LS7000). Total counts, after correction for dilution and quenching, were converted to picograms and the accumulation of C $^{14}$-TPP was expressed in picograms per milligram. An identical protocol was carried out in other series of experiments, except that the adrenal gland was always pretreated with a modified Krebs solution (i.e., hypertonic or $55 \mathrm{~mm} \mathrm{~K}$, etc.) for $10 \mathrm{~min}$ prior to switchover to modified Krebs solution containing $\mathrm{C}^{14}$-TPP.

\section{$\mathrm{Ca}^{45}$ accumulation}

The adrenal gland was perfused with Krebs solution for $60 \mathrm{~min}$ and then the medium was changed for $10 \mathrm{~min}$ to Krebs solution containing $0.5 \mu \mathrm{g} / \mathrm{ml} \mathrm{Ca}^{45}$ (as $\mathrm{Ca}^{45} \mathrm{Cl}_{2}$, specific activity $23.69 \mathrm{mCi} / \mathrm{mg}$; New England Nuclear). The gland was perfused for $30 \mathrm{~min}$ with radiolabel-free Krebs solution to wash out the extracellular $\mathrm{Ca}^{45}$. In 4 experiments, the radioactivity in wash fluid was determined from the point of termination of perfusion with $\mathrm{Ca}^{45}-\mathrm{Krebs}$ solution for up to $30 \mathrm{~min}$. The first $5 \mathrm{~min}$ sample contained $96+0.04 \times 10^{4} \mathrm{cpm}$, whereas 20 and 25 min samples contained $0.023 \pm 0.005 \times 10^{4}$ and $0.021 \pm 0.003 \times 10^{4} \mathrm{cpm}$, respectively.

Basically the same protocol was followed in various types of experiments, except that $\mathrm{Ca}^{45}$ was introduced at different times after initial perfusion of the adrenal gland with modified Krebs solution, as described in the text. Then the gland was quickly removed and the medulla was separated and rinsed several times with ice-cold Krebs solution. The medulla was gently blotted, weighed, and then homogenized in 1 $\mathrm{ml}$ of $0.1 N$ perchloric acid in a glass homogenizer. The homogenate was centrifuged $(2000 \mathrm{rpm})$ for $5 \mathrm{~min}$, and radioactivity in $0.5 \mathrm{ml}$ supernatant was measured in a liquid scintillation counter (Beckman Model LS7000). Appropriate corrections for volume changes and quenching have been made. The amounts of $\mathrm{Ca}^{45}$ accumulated in the control gland in each group of experiments were subtracted from those in the experimental gland to obtain the net accumulation of $\mathrm{Ca}^{45}$; they are expressed as picograms per milligram of the wet weight of the adrenal medulla.

\section{Measurement of $C A$}

$\mathrm{CA}$ content in the perfusate was assayed directly by the fluorometric method of Anton and Sayre (1962). Because of low sample blanks, it was possible to detect up to $5 \mathrm{ng}$ in a $2 \mathrm{ml}$ perfusate without further concentration by alumina treatment.

CA content of the adrenal medulla was measured in some experiments. After removal of the adrenal gland the medulla was separated, weighed, and homogenized in $0.5 \mathrm{ml}$ of $0.1 \mathrm{~N}$ perchloric acid. The homogenate was centrifuged and an aliquot $(0.2 \mathrm{ml})$ was diluted $100 \times$ with ice-cold distilled water; $0.2 \mathrm{ml}$ of this fluid was used for CA determination, using the method of Anton and Sayre (1962). Total tissue content was expressed as micrograms per milligram of adrenal medulla. The content of CA in the perfusate and the adrenal medulla was calculated in terms of the epinephrine base.

\section{Lactate dehydrogenase}

Lactate dehydrogenase in the perfusate and the adrenal medulla was analyzed by the method of Wroblewski and LaDue (1955), using Sigma Kit No. 340UV. One milliliter of the perfusate was used for the assay. In the case of the adrenal medulla, the tissue was homogenized in $1 \mathrm{ml}$ of ice-cold $0.1 \mathrm{M}$ phosphate buffer, centrifuged, and the supernatant was divided into 2 portions. One part was diluted $10 \times$ with phosphate buffer and used for determination of lactate dehydrogenase. The other portion was first diluted $10 \times$ with $0.5 \mathrm{~N}$ perchloric acid, then diluted $10 \times$ with water and used for CA estimation.

All data were presented as means with standard errors, and differences were compared using Student's $t$ test.

\section{Drugs and chemicals used}

The following drugs and chemicals were used in these experiments: nicotine (Aldrich Chemical Corp., Milwaukee, WI); TTX (CalbiochemBehring Corp., La Jolla, CA); atropine sulfate, hexamethonium bromide (Sigma Chemical Corp., St. Louis, MO); choline chloride (Eastman Kodak Company, Rochester, NY); sodium chloride and sucrose (Fisher Scientific Corp., Fairlawn, NJ); arginine hydrochloride (Nutritional Biochem, Cleveland, $\mathrm{OH}$ ); and sodium thiocyanate (Merck and Company, Rahway, NJ).

\section{Results}

\section{Effect of hypertonic Krebs solution on spontaneous and stimulation-evoked secretion of $C A$}

The spontaneous secretion of CA from the rat adrenal gland perfused with Krebs bicarbonate solution remained steady and at a very low level over prolonged periods of time $(15 \pm 2 \mathrm{ng}$ at $60 \mathrm{~min}$ and $10 \pm 3 \mathrm{ng}$ at $240 \mathrm{~min} ; n=6$ ). If the adrenal gland was perfused with hypertonic Krebs solution (made by adding $156 \mathrm{~mm} \mathrm{NaCl}$ ) for several hours, there was no change in the normal pattern of spontaneous secretion of CA $(15 \pm 10$ ng vs $10 \pm 2 \mathrm{ng} ; n=13$ ).

In subsequent experiments the effect of hypertonic medium on stimulation-evoked secretion of CA was examined. Secretion of CA evoked by nerve stimulation $(5 \mathrm{~Hz}$ for $1 \mathrm{~min}$ ) or $2 \mu \mathrm{g}$ nicotine in hypertonic medium was almost identical to that obtained in normal medium in the same adrenal gland (142 \pm $11 \mathrm{vs} 155 \pm 18 \mathrm{ng}$ for $5 \mathrm{~Hz}$ and $139 \pm 11$ vs $134 \pm 16 \mathrm{ng}$ for nicotine; $n=3$ ). Each sample was collected for $5 \mathrm{~min}$.

\section{Secretion of $C A$ after exposure of the adrenal gland to hypertonic Krebs solution}

If the perfusion medium was switched to normal Krebs solution after $1 \mathrm{hr}$ perfusion with hypertonic medium, there was a massive secretory response within the first $5 \mathrm{~min}$ period (Fig. 1). The secretion remained at that level (about $400 \mathrm{ng}$ ) for the next $10 \mathrm{~min}$ and gradually declined to about $100 \mathrm{ng}$ after $30 \mathrm{~min}$. The secretion remained near the $50 \mathrm{ng}$ level even $90 \mathrm{~min}$ after switchover to the normal medium. As is shown in Figure 1, after perfusion with Krebs solution for about $2 \mathrm{hr}$, the secretion of CA approached the basal control levels. 


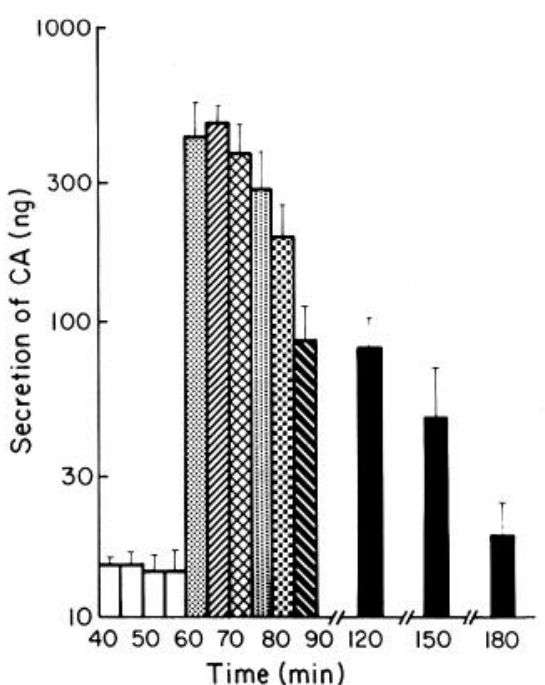

Figure 1. Secretion of CA in normal Krebs solution following exposure to hypertonic medium. After $30 \mathrm{~min}$ perfusion with Krebs solution, the medium was changed to hypertonic Krebs solution ( $156 \mathrm{~mm} \mathrm{NaCl}$ added to Krebs solution) for $60 \mathrm{~min}$. Four $5 \mathrm{~min}$ samples within the last 20 min of perfusion with hypertonic solution were collected for CA assay (open columns). After $60 \mathrm{~min}$, the perfusion medium was changed to normal Krebs solution and 5 min serial samples were collected immediately after the switchover of the solution, up to $30 \mathrm{~min}$ (shaded columns). Additional samples were collected, as shown, at various time intervals. Each column represents a mean of 4-7 experiments. Vertical lines, SEM.

\section{Secretion of CA after perfusion with hypertonic Krebs} solution made by the addition of different agents

Results of these experiments are summarized in Table 1. As stated above, perfusion of the adrenal gland with Krebs solution throughout the experiment did not modify the secretion. However, $1 \mathrm{hr}$ exposure to hypertonic Krebs solution made by the addition of either $156 \mathrm{~mm}$ sodium chloride, choline chloride, arginine hydrochloride, or sodium thiocyanate, followed by perfusion with normal Krebs solution, resulted in an enhanced secretion of CA. Hypertonic solution made with $312 \mathrm{~mm}$ sucrose was also effective in inducing the secretion. It should be pointed out that perfusion of the adrenal gland with $156 \mathrm{~mm}$ of different agents in Krebs solution did not affect the basal secretion of CA.

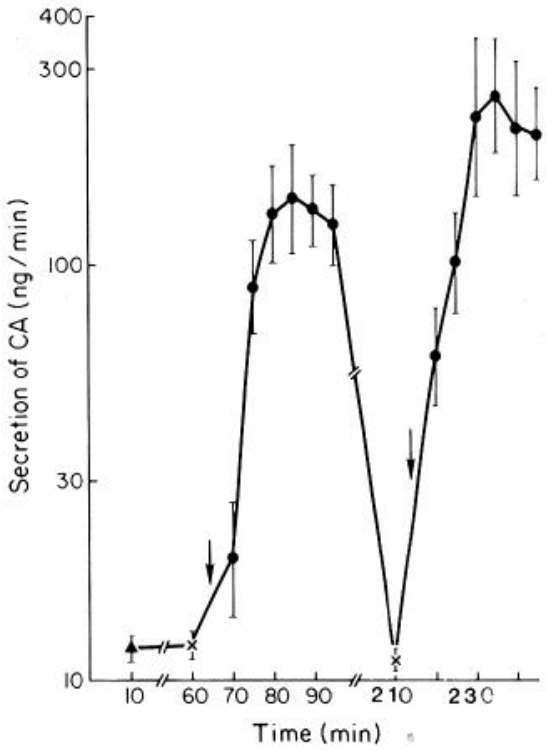

Figure 2. Secretion of CA after second exposure to hypertonic medium. The experimental protocol was identical to that described in Figure 1, except that $156 \mathrm{~mm}$ sodium thiocyanate was used to make hypertonic Krebs solution. Secretion of CA was determined first in normal Krebs solution $(\boldsymbol{\Delta})$ and then in hypertonic medium after $60 \mathrm{~min}$ perfusion $(\times)$. A series of samples were collected as shown $(\bullet)$ when the medium was switched to normal Krebs (at the arrow). After 150 min, the adrenal gland was reperfused with hypertonic Krebs solution for $60 \mathrm{~min}$, a sample was removed $(\times)$, and the medium was switched over to normal Krebs (at the arrow), and another series of samples were collected at various points $(\bullet)$ for CA analysis. Each sample was collected for $5 \mathrm{~min}$. Each point is a mean of 3 observations. Vertical lines, SEM.

\section{Reproducibility of CA secretion after repeated exposure to hypertonic Krebs solution}

Because of the unexpected and unusual nature of the secretion of CA obtained following, but not during, exposure to the hypertonic medium, it was essential to establish that such a secretion was not a result of tissue damage. Two types of experiments were designed to examine this point. In one, the ability of the adrenal gland to secrete CA in response to electrical stimulation $(10 \mathrm{~Hz})$ was compared in normal Krebs solution

Table 1. Catecholamine secretion after perfusion with hypertonic solution made by addition of various agents to the Krebs solution

\begin{tabular}{llll} 
& \multicolumn{3}{l}{ Secretion of catecholamines (ng) } \\
\cline { 2 - 4 } Type of hypertonic Krebs solution & Krebs & Hypertonic & Krebs \\
\hline Regular Krebs $(119 \mathrm{~mm} \mathrm{NaCl})$ & $15 \pm 10$ & $25 \pm 15$ & \multicolumn{1}{c}{ Krebs } \\
$+156 \mathrm{~mm}$ sodium chloride & $25 \pm 5$ & $15 \pm 5$ & $460 \pm 40$ \\
$+156 \mathrm{~mm}$ choline chloride & $20 \pm 10$ & $35 \pm 15$ & $370 \pm 45$ \\
$+156 \mathrm{~mm}$ arginine hydrochloride & $10 \pm 5$ & $10 \pm 10$ & $150 \pm 40$ \\
$+156 \mathrm{~mm}$ sodium thiocyanate & $20 \pm 5$ & $20 \pm 5$ & $270 \pm 85$ \\
$+156 \mathrm{~mm}$ sucrose & $25 \pm 10$ & $15 \pm 10$ & $10 \pm 10$ \\
$+312 \mathrm{~mm}$ sucrose & $5 \pm 5$ & $20 \pm 5$ & $515 \pm 94$
\end{tabular}

After 30 min perfusion with normal Krebs solution, one 5 min sample was collected, and then the adrenal gland was perfused for $1 \mathrm{hr}$ with Krebs solution made hypertonic by addition of 156 and $312 \mathrm{~mm}$ of the agents shown above. A $5 \mathrm{~min}$ sample during the last $60 \mathrm{~min}$ perfusion period with hypertonic Krebs solution was analyzed, and then the medium was changed to normal Krebs solution. Immediately after the switchover, a 5 min sample was removed. CA analyzed in each $5 \mathrm{~min}$ sample were expressed as total secretion in nanograms. Each value is the mean of 3-12 observations. 

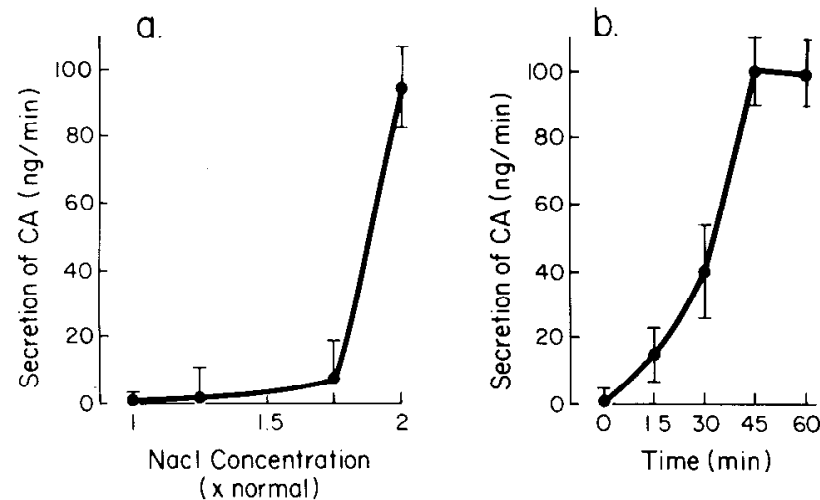

Figure 3. Degree of hypertonicity and time of exposure to hypertonic solution essential to initiate CA secretion. $a$, Adrenal glands were perfused with Krebs solution to which increasing amounts of $\mathrm{NaCl}$ were added, to a final concentration of $275 \mathrm{~mm}$. The adrenal gland was perfused with each hypertonic solution for $1 \mathrm{hr}$. The increasing strengths of the hypertonic solutions were tested in the same gland after the basal secretion had reached a low control valuc (about 5-10 $\mathrm{ng} / 5 \mathrm{~min}$ sample). $b$, Hypertonic solution ( $156 \mathrm{~mm}$ sodium chloride added to Krebs solution) was used to perfuse the adrenal gland for varying periods of time, as shown, and then the medium was switched over to normal Krebs solution. The same adrenal gland was used to test various exposure periods after basal secretion was restored toward control level (see above). In both series of experiments, CA secretion within the first $10 \mathrm{~min}$ of switchover was measured, and these amounts are expressed as $\mathrm{ng} / \mathrm{min}$. Each point is a mean of $4-5$ experiments. Vertical lines, SEM.

before and after exposing the adrenal gland to the hypertonic solution. In 3 experiments it was found that the secretory response of the adrenal gland after hypertonic challenge $(60 \mathrm{~min}$ of hypertonic medium followed by $150 \mathrm{~min}$ perfusion with normal medium) was still $81 \pm 16 \%$ of the control value obtained in Krebs solution at the start of the experiment. Similarly, nicotine-evoked secretion was unaffected by treatment of the adrenal gland with hypertonic Krebs solution followed by $150 \mathrm{~min}$ perfusion with normal Krebs solution $(267 \pm 17 \mathrm{ng}$ vs $252 \pm$ $29 \mathrm{ng}$ ).

In another series of experiments, viability was tested by perfusing the same adrenal gland for the second time with hypertonic solution and then examining the secretion during perfusion with normal Krebs solution. The results of such experiments are shown in Figure 2. When the secretory response approached essentially control value after the first exposure to hypertonic and then to normotonic media, the adrenal gland was reperfused with $156 \mathrm{~mm}$ sodium thiocyanate-Krebs solution for $1 \mathrm{hr}$, and then with normal Krebs solution. As is shown in Figure 2, the secretion of CA increased soon after the return to normal medium and remained elevated over a $10 \mathrm{~min}$ period. The adrenal gland was not only capable of responding to a second challenge of hypertonic medium but, in fact, the secretion of CA was even further enhanced after the second exposure to the hypertonic medium.

\section{Degree of hypertonicity and time of exposure to hypertonic solution required to evoke secretion of $C A$}

Sodium chloride concentration of the Krebs solution was increased in separate experiments from a control value of $119 \mathrm{mM}$ to $275 \mathrm{~mm}$ to study the effect of gradual increases in the degree of tonicity on the secretion of CA. As is shown in Figure $3 a$, a significant increase $(p<0.05)$ in CA secretion occurred after perfusion of the adrenal gland with $1.75 \times$ the normal concentration of sodium chloride in the Krebs solution. The secretory response increased sharply (over 13-fold) when the sodium chloride concentration was raised to more than 2 -fold the normal concentration $(119+156 \mathrm{~mm})$.

In another series of experiments, $275 \mathrm{~mm}$ sodium chlorideKrebs solution was used to perfuse the adrenal gland for varying periods of time, and then secretion was examined in normal Krebs solution to establish the time course (Fig. $3 b$ ). A $15 \mathrm{~min}$ exposure of the adrenal gland to hypertonic solution was sufficient to evoke significantly enhanced secretion during perfusion with normal Krebs solution. A further increase in the exposure period to 30 and $45 \mathrm{~min}$ led to a still enhanced secretion of CA. Secretion was not further increased if the exposure period was increased to $60 \mathrm{~min}$.

\section{Effect of various procedures on the secretion of CA evoked after exposure to a hypertonic medium}

The secretion of CA obtained after treatment of the adrenal gland with hypertonic medium could be a result of activation of nicotinic and muscarinic receptors of the chromaffin cells by ACh released from the splanchnic nerve terminals during switchover from hypertonic to normotonic media. To determine the role of the presynaptic component in the present phenomenon, several types of experiments were performed. In one, identical experiments - as described in Figure 1-were carried out in chronically splanchnectomized adrenal glands. As is shown in Table 2 , the secretory response normally observed after perfusion with hypertonic Krebs solution, followed by normal me-

Table 2. Effects of splanchnectomy, TTX, and cholinergic antagonists on catecholamine secretion evoked after exposure to hypertonic Krebs solution

\begin{tabular}{|c|c|c|c|}
\hline \multirow[b]{2}{*}{ Treatment } & \multicolumn{3}{|c|}{ Secretion of catecholamines (ng) ${ }^{a}$} \\
\hline & Krebs & $\begin{array}{l}\text { Hypertonic } \\
\text { Krebs }\end{array}$ & Krebs \\
\hline None $^{b}$ & $15 \pm 5$ & $15 \pm 5$ & $460 \pm 10$ \\
\hline Splanchnectomy ${ }^{c}$ & $5 \pm 1$ & $20 \pm 5$ & $505 \pm 15^{f}$ \\
\hline Tetrodotoxin $^{d}(1 \mu \mathrm{M})$ & $5 \pm 1$ & $5 \pm 2$ & $470 \pm 120^{\prime}$ \\
\hline Atropine $(1.5 \mu \mathrm{M})+$ hexamethonium $(138 \mu \mathrm{M})^{c}$ & $12 \pm 5$ & $7 \pm 1$ & $370 \pm 55^{r}$ \\
\hline \multicolumn{4}{|l|}{${ }^{a}$ Each value represents a mean of $3-7$ experiments. } \\
\hline \multicolumn{4}{|c|}{$\begin{array}{l}{ }^{b} \text { Experimental protocol identical to that in Table } 1 . \text { The first row of values represents a "control" for the remaining } \\
\text { types of experiments carried out after different treatments. }\end{array}$} \\
\hline \multicolumn{4}{|c|}{ Left adrenal gland was surgically denervated and used for perfusion $2-3$ weeks after the operation. } \\
\hline \multicolumn{4}{|c|}{$\begin{array}{l}\text { TTX was added during the last } 30 \text { min perfusion with hypertonic Krebs solution and also was present when the medium } \\
\text { was switched over to the Krebs solution. }\end{array}$} \\
\hline
\end{tabular}




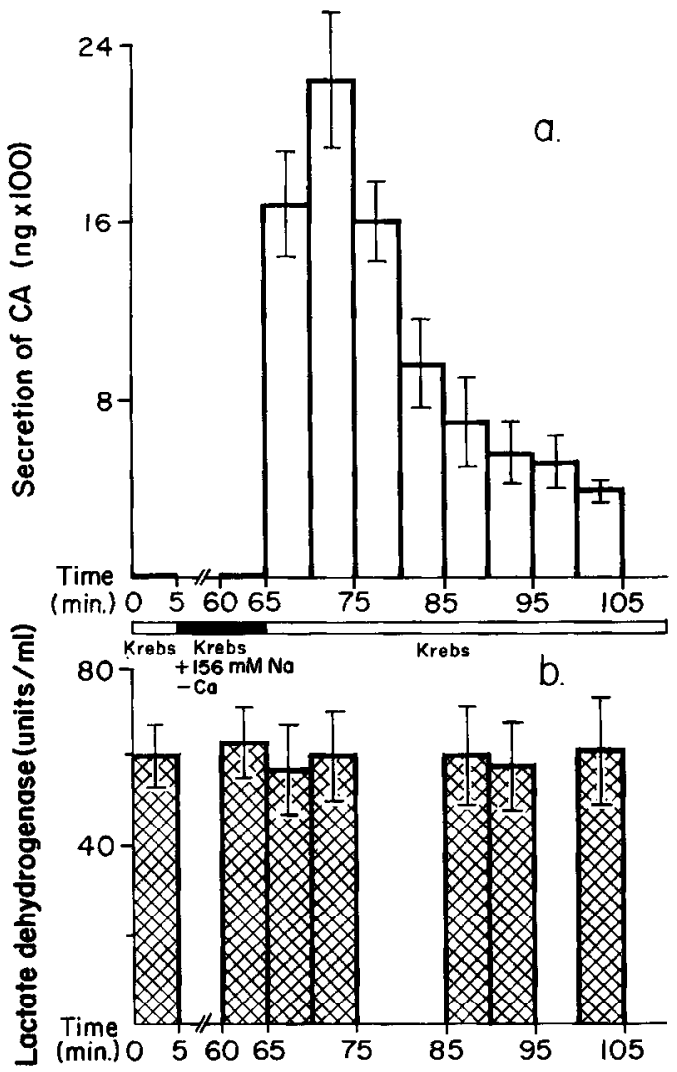

Figure 4. Secretion of CA after switchover from Ca-free and hypertonic Krebs solution to normal medium. a, After 30 min perfusion with Krebs solution, one $5 \mathrm{~min}$ sample $(0-5 \mathrm{~min})$ was collected and the medium was changed to Ca-free, $275 \mathrm{~mm} \mathrm{NaCl}$-Krebs solution for 60 min, and one 5 min sample collected. Then the medium was changed to normal Krebs solution, and 5 min serial samples were collected immediately after the switchover of the solution, up to $40 \mathrm{~min}$. All samples were assayed for CA. $b$, Some of the samples derived from the experiment $(a)$ were also assayed for lactate dehydrogenase activity, as described in Materials and Methods. Each column represents a mean of 4-13 experiments. Vertical lines, SEM.

dium, remained unaffected by degeneration of splanchnic nerve terminals. The success of splanchnectomy was attested to in each adrenal gland at the beginning of the experiment by applying transmural stimulation, which is known to evoke release mainly by activation of the splanchnic nerves (Wakade, $1981 \mathrm{~b})$. In none of the splanchnectomized adrenal glands used in the present study did $10 \mathrm{~Hz}$ stimulation causc a significant secretion of CA in comparison to that in the innervated glands (data not shown).

In other experiments, adrenal glands were perfused with hypertonic medium containing a combination of atropine $(1.5 \mu \mathrm{M})$ and hexamethonium $(138 \mu \mathrm{M})$ in order to inactivate muscarinic and nicotinic receptors, respectively (Wakade and Wakade, 1983). When this medium was changed over to normal Krebs solution containing atropine plus hexamethonium, the typical secretory response was still present (Table 2).

The possibility was considered that during switchover from hypertonic to normotonic solution there may be repetitive firing along the splanchnic nerves (and chromaffin cells) that is mainly responsible for the prolonged secretion of CA seen after such a maneuver. However, treatment of the adrenal gland with $1 \mu \mathrm{M}$ TTX failed to modify the secretory response seen after switchover from hypertonic to normal Krebs solution (Table 2).

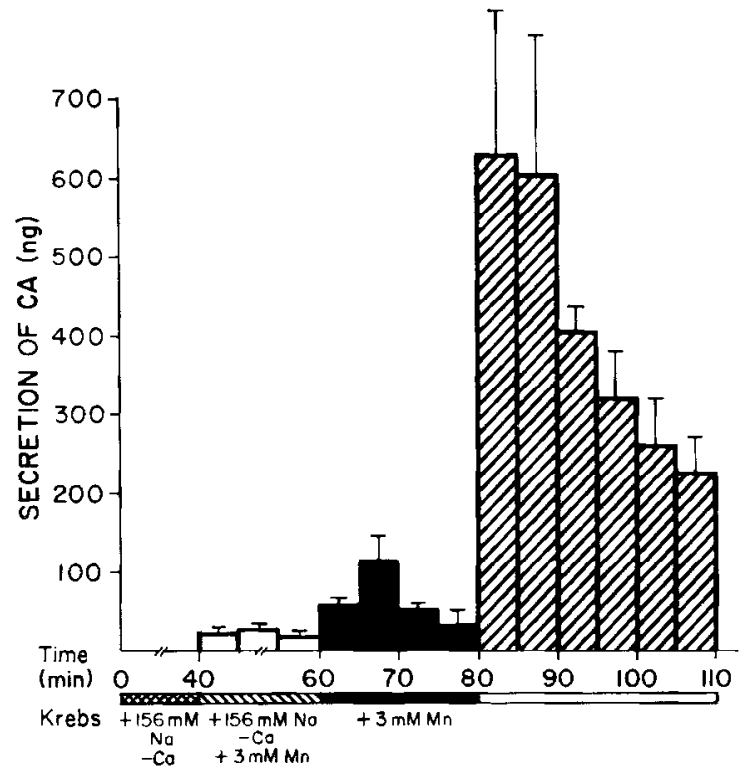

Figure 5. Effect of $\mathrm{Mn}$ on the explosive secretion of CA. After $30 \mathrm{~min}$ perfusion with Krebs solution, the medium was changed to Ca-free and $275 \mathrm{~mm} \mathrm{NaCl}$-Krebs solution for $\mathbf{4 0} \mathrm{min}$, and $3 \mathrm{~mm}$ Mn was added to the same medium for an additional $20 \mathrm{~min}$. Then the medium was changed to Krebs solution containing $3 \mathrm{mM} \mathrm{Mn}$, and 5 min serial samples were collected immediately after the switchover of solutions. After $20 \mathrm{~min}$, the medium was changed to regular Krebs solution and $5 \mathrm{~min}$ samples were collected immediately and for up to $30 \mathrm{~min}$. For this series of experiments, Ca-free Krebs solution was prepared by adding $11 \mathrm{~mm}$ IIEPES to the solution, in the place of $\mathrm{NaHCO}_{3}$ and $\mathrm{KH}_{2} \mathrm{PO}_{4}, \mathrm{pH}$ was adjusted to 7.4 , and the solution was bubbled with $100 \% \mathrm{O}_{2}$. To this solution, $2.5 \mathrm{~mm} \mathrm{CaCl}$ was added to obtain normal Krebs solution, and $156 \mathrm{mM} \mathrm{NaCl}$ was added as required. Each bar represents a mean of 4 experiments. Vertical lines, SEM.

The obligatory role of calcium in the secretion of $C A$ evoked after exposure to hypertonic Krebs solution

Although a number of the above-described experiments revealed that the secretion of CA obtained from the adrenal medulla after exposure to hypertonic solution was not a result of tissue damage, it was essential to know whether Ca was needed in this secretory process. Therefore, several types of experiments were carried out to cstablish the role of $\mathrm{Ca}$ in the secretion evoked after hypertonic treatment.

As is shown in Figure $4 a$, if $\mathrm{Ca}$ was omitted during $1 \mathrm{hr}$ perfusion with hypertonic medium $(275 \mathrm{~mm} \mathrm{NaCl}-\mathrm{Krebs}$ solution) and introduced in a normotonic solution, the secretion of CA increased from 10 to about $4000 \mathrm{ng}$ within the first 10 min, and gradually declined to $400 \mathrm{ng} 40 \mathrm{~min}$ after the switchover. The secretory response persisted for another $40 \mathrm{~min}$ (data not shown). Because of the explosive nature of the secretion observed in these experiments, it was essential to rule out the possibility of medullary cell lysis. Therefore, lactate dehydrogenase content of the adrenal perfusates was estimated during perfusion with different types of solution. Figure $4 b$ shows that the enzyme content remained unchanged during the entire course of the experiment.

Two types of control experiments were carried out for the above series of experiments. In one series, the adrenal gland was perfused with Ca-free Krebs solution for $1 \mathrm{hr}$ and then switched over to Krebs solution. There was no massive increase in the secretion of CA (13 $\pm 6 \mathrm{ng}$ vs $21 \pm 8 \mathrm{ng} ; n=4)$. In the other, the adrenal gland was perfused with $275 \mathrm{~mm} \mathrm{NaCl}$ and $\mathrm{Ca}$-free Krebs solution for $1 \mathrm{hr}$ and then changed over to 275 
a

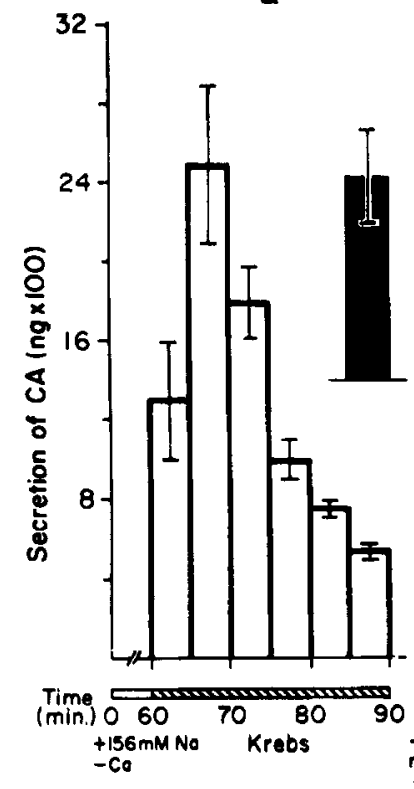

b

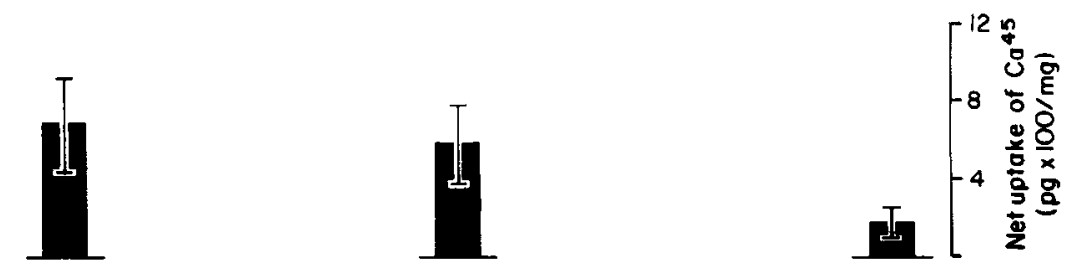

Figure 6. Accumulation of $\mathrm{Ca}^{45}$ and secretion of $\mathrm{CA}$ at various times after switchover from Ca-free and hypertonic Krebs to normal Krebs solution. a, Adrenal gland was perfused with Ca-free and hypertonic Krebs solution for $1 \mathrm{hr}$ and then with regular Krebs, as described in Figure 5. In another series of experiments, after perfusion of the adrenal gland with Ca-free and $275 \mathrm{~mm} \mathrm{NaCl}-\mathrm{Krebs}$ solution, the medium was switched to Ca-free Krebs solution (normotonic) for $30(b), 60(c)$ and $120(d)$ min prior to perfusion with Krebs solution. Six 5 min samples were collected in each group to determine the secretion of CA. Each bar is a mean of 3-16 experiments. In another group of adrenal glands, the entire protocol ( $a-d)$ was repeated as described above, except that normal Krebs solution (after perfusion with hypertonic medium) contained $0.5 \mu \mathrm{g} / \mathrm{ml} \mathrm{Ca}{ }^{45} \mathrm{Cl}_{2}$ for $10 \mathrm{~min}$, which was washed for $30 \mathrm{~min}$ prior to removal of adrenal medulla for estimation of radioactivity due to $\mathrm{Ca}^{45}$ (solid bar), as described in Materials and Methods. In still another group, the above protocol was repeated, except that the adrenal glands were perfused with Ca-free Krebs solution (normotonic) for $60 \mathrm{~min}$ prior to switchover to $\mathrm{Ca}$-free normotonic solution for various periods of time prior to 10 min exposure to Ca ${ }^{45}$. These control experiments were performed to determine the accumulation of $\mathrm{Ca}^{45}$ under "nonstimulation condition." The amounts of $\mathrm{Ca}^{45}$ accumulated in these control experiments $(229 \pm 42 \mathrm{pg} / \mathrm{mg} ; n=8)$ have been subtracted from the above group to obtain net uptake of Ca ${ }^{45}$ for each group. In still 7 other glands, the uptake of $\mathrm{Ca}^{45}$ was examined 30 min after perfusion with regular Krebs solution $(207 \pm 40 \mathrm{pg} / \mathrm{mg} ; n=7)$. There was no significant difference between these two values. Each solid bar is a mean of 3-5 experiments. Vertical lines, SEM.

mM NaCl-Krebs solution. This change also did not result in a marked increase in the secretion of CA ( $74 \mathrm{ng}$ vs $17 \pm 9 \mathrm{ng}$; $n=3$ ).

Whether the explosive secretion seen in Figure 4 required $\mathrm{Ca}$ when the perfusion medium was changed from hypertonic and $\mathrm{Ca}-$ free to $\mathrm{Ca}$-containing Krebs solution was tested in the next series of experiments.

\section{Antagonism by manganese}

Earlier, it was shown that Mn blocked the Ca-dependent secretion of $\mathrm{CA}$ evoked by $\mathrm{ACh}$ and splanchnic nerve stimulation in the rat adrenal gland (Wakade, 1981a). Therefore, the effects of $3 \mathrm{~mm} \mathrm{Mn}$ were investigated in the present study; the results are shown in Figure 5. During the final $15 \mathrm{~min}$ of $1 \mathrm{hr}$ perfusion with hypertonic and $\mathrm{Ca}$-free $\mathrm{Krebs}$ solution, $\mathrm{Mn}$ was introduced into the medium, and was also present when the solution was changed to normal Krebs solution. As is shown in Figure 5, Mn prevented the secretion of CA during switchover. Figure 5 also shows that, as Mn was being washed out, the secretory response began to appear at a significant level in the same adrenal gland.

\section{Secretion of $C A$ at various times after exposure to calcium}

In another series of experiments, $\mathrm{Ca}$ was presented at various time intervals after the adrenal gland was exposed to $\mathrm{Ca}$-free, hypertonic medium and then to $\mathrm{Ca}$-free, normotonic medium. The results of these experiments are summarized in Figure 6. Immediate exposure to $\mathrm{Ca}$ after the switchover produced a massive secretory response (Fig. $6 a$ ), as has also been found in previous experiments (Fig. 4). However, if $\mathrm{Ca}$ was presented after $30 \mathrm{~min}$, there was still a large increase in the secretion of CA (from essentially undetectable levels to $800 \mathrm{ng}$ in $5 \mathrm{~min}$; Fig. 6 b). If introduction of $\mathrm{Ca}$ was delayed as much as $60 \mathrm{~min}$ after the gland had been perfused with $\mathrm{Ca}$-free and hypertonic solution for $1 \mathrm{hr}$, and then another $60 \mathrm{~min}$ with Ca-free Krebs (normotonic) solution, a marked increase in the secretion occurred (Fig. $6 c$ ). Finally, as shown in Figure $6 d$, significant quantities of CA were secreted even 120 min after presentation of $\mathrm{Ca}$ to the gland.

\section{Ca $a^{45}$ accumulation}

To determine if the secretion of CA obtained in the above series of experiments was a result of the influx of $\mathrm{Ca}$, a study was made of the accumulation of $\mathrm{Ca}^{45}$ at various times, as has been described in previous experiments. The results are included in Figure 6. The accumulation of $\mathrm{Ca}^{45}$ increased 6 -fold over the control when $\mathrm{Ca}^{45}$ was presented immediately after the switchover from hypertonic to normotonic solution (Fig. $6 a$ ). Although the accumulation of $\mathrm{Ca}^{45}$ gradually declined with an increase in the time interval between presentation of $\mathrm{Ca}^{45}$ and termination of perfusion with $\mathrm{Ca}$-free and hypertonic solution, a significant accumulation of $\mathrm{Ca}^{45}$ was detected even $2 \mathrm{hr}$ after the change (Fig. 6d).

Assessment of membrane potential by $C^{14}-T P P$ accumulation Changes in the membrane potential of the perfused adrenal medulla were monitored by measuring the accumulation of $\mathrm{C}^{14}$ TPP. Several investigators have shown that the accumulation of $\mathrm{C}^{14}$-TPP decreases when the cell membrane depolarizes, and 

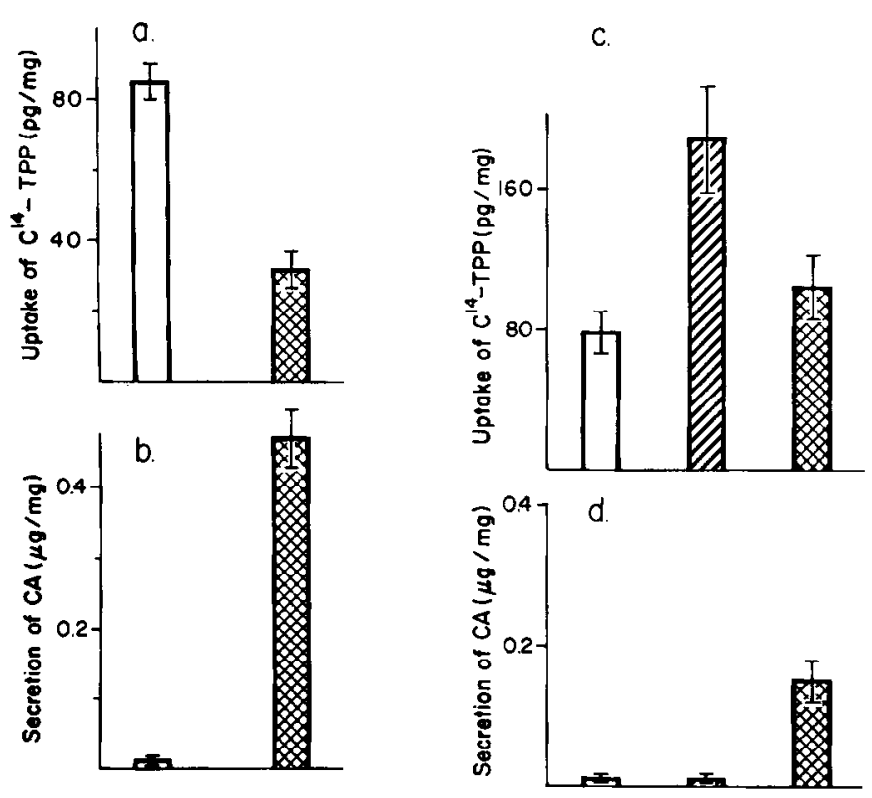

Figure 7. C14-TPP accumulation of the adrenal medulla perfused with normal and excess K-Krebs solution. $a$, After $30 \mathrm{~min}$ perfusion with Krebs solution, the medium was changed over to Krebs solution containing $\mathrm{C}^{14}$-TPP for $45 \mathrm{~min}$, and then the adrenal gland was perfused with ice-cold Krebs solution for $10 \mathrm{~min}$. The adrenal medulla was removed for analysis of radioactivity due to C14-TPP (open bar), as described in Materials and Methods. In another group, the identical protocol was carried out except that $55 \mathrm{~mm} \mathrm{~K}$ was present $10 \mathrm{~min}$ before and during $45 \mathrm{~min}$ perfusion with $\mathrm{C}^{14}-\mathrm{TPP}$ Krebs solution (hatched $b a r) . b$. The above 45 min samples from each group were analyzed for $\mathrm{CA}$ and expressed as $\mu \mathrm{g} / \mathrm{mg}$ of adrenal weight. $c$, $\mathrm{C}^{14}$-TPP accumulation in the adrenal medulla perfused with hypertonic and then hypertonic followed by normotonic solutions. The experimental protocol was identical to that described in $a$. After initial perfusion with Krebs solution, the medium was changed over to $275 \mathrm{~mm} \mathrm{NaCl}$-Krebs solution for 30 min, then to the same solution containing $C^{14}-T P P$ for $45 \mathrm{~min}$, and washed with ice-cold Krebs solution, as above, prior to removal of the adrenal medulla to determine C 14 -TPP content (hatched bar). In another group the adrenal gland was perfused with $275 \mathrm{mM} \mathrm{NaCl}-\mathrm{Krebs}$ solution for $60 \mathrm{~min}$; then the medium was changed over to Krebs solution containing $\mathrm{C}^{14}$-TPP for $45 \mathrm{~min}$, followed by a $10 \mathrm{~min}$ wash prior to removal of the adrenal medulla (cross-hatched bar). $d$, The above 45 min samples from each group were analyzed for CA. Each bar is a mean of 5 observations. Vertical lines, SEM.

increases when the membrane hyperpolarizes (Kiefer et al., 1980; Lichtshtein et al., 1979; Wakade and Wakade, 1982b; Wakade et al., 1983; Wastek et al., 1981).

First, the applicability of the technique in assessing the membrane potential by using C $^{14}-$ TPP was determined by comparing the accumulation of $\mathrm{C}^{14}$-TPP in the adrenal medulla perfused with normal medium with the accumulation of C14-TPP in 55 mM K-Krebs solution. Figure $7 a$ shows that the acumulation of $\mathrm{C}^{14}$-TPP was markedly reduced $(63 \%)$ in $55 \mathrm{~mm} \mathrm{~K}-\mathrm{Krebs}$ solution, which was associated with a marked increase in the secretion of CA $(0.02-0.45 \mu \mathrm{g} / \mathrm{mg}$; Fig. $7 b)$.

In contrast to the accumulation of C ${ }^{14}-$ TPP (about $80 \mathrm{pg} / \mathrm{mg}$ ) in the adrenal medulla perfused with normal medium, the accumulation increased to about $190 \mathrm{pg} / \mathrm{mg}$ after perfusion with $275 \mathrm{~mm} \mathrm{NaCl}-$ Krebs solution. After switching to hypertonic medium, if the adrenal gland was perfused with normotonic medium, the accumulation of C14-TPP was reduced to $104 \mathrm{pg}$ / $\mathrm{mg}$ (Fig. $7 \mathrm{c}$ ). In these experiments the perfusates, before and during C $\mathrm{C}^{14}$-TPP exposure, were assayed for CA; the results are shown in Figure $7 d$. The amounts of CA secreted in various media werc just as expected from the results obtained in previous experiments.

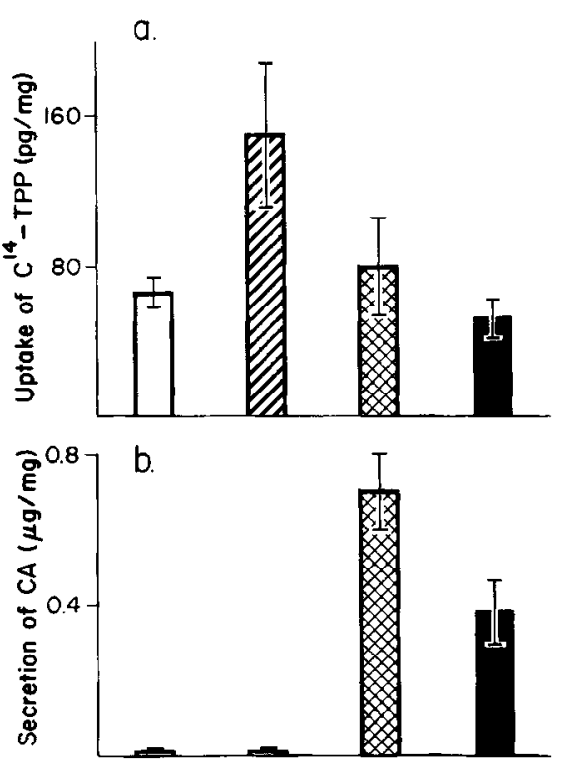

Figure 8. C C $^{14}$-TPP accumulation in the adrenal medulla perfused with $\mathrm{Ca}-$ free and $275 \mathrm{~mm} \mathrm{NaCl}$-Krebs solution, followed by Krebs solution. $a$, The experimental protocol (for the first 3 bars, starting from the left) was identical to that described in Figure $7 c$, except that hypertonic solution was deprived of $\mathrm{Ca}$, as described in Figure 4. The same figure also shows the inhibitory effect of $30 \mathrm{~mm} \mathrm{~K}$ on the accumulation of $\mathrm{C}^{14}$-TPP and CA secretion obtained after switchover of Ca-free and hypertonic solution to normotonic solution. After initial perfusion with normal medium, the adrenal gland was perfused with Ca-free and 275 mM NaCl-Krebs solution for $50 \mathrm{~min}$, followed by $30 \mathrm{~mm} \mathrm{~K}$-, Ca-free, and $275 \mathrm{~mm} \mathrm{NaCl}$-Krebs solution for $10 \mathrm{~min}$ and then with $30 \mathrm{~mm} \mathrm{~K}-$ Krebs containing $C^{14}$-TPP for $45 \mathrm{~min}$, washed for $10 \mathrm{~min}$ prior to removal of the adrenal medulla (solid bar in $a$ ). $b$, The above $45 \mathrm{~min}$ samples from each group were analyzed for CA. In this series of experiments, $30 \mathrm{~mm} \mathrm{~K}$ was added to Krebs solution without removing the equivalent amounts of $\mathrm{NaCl}$. Each bar is the mean of 4 experiments. Vertical lines, SEM.

Identical results were obtained in another group of experiments, in which $\mathrm{C}^{14}$-TPP accumulation was determined in the adrenal medulla perfused with $275 \mathrm{~mm} \mathrm{NaCl}$ and Ca-free medium, and after switching from this medium to normal Krebs solution (Fig. 8).

If the explosive secretion of CA was a result of change in the membrane potential (i.e., "depolarization"), then the presence of excess $K$ should interfere with the secretion during changeover from hypertonic to normotonic solution. The results of experimental studies are shown in Figure 9. As expected, perfusion with $30 \mathrm{mM}$ K-Krebs solution caused a good secretion of CA compared to the spontaneous secretion. The secretion was essentially zero when $\mathrm{Ca}$ was omitted from $275 \mathrm{mM} \mathrm{NaCl}$ and $30 \mathrm{~mm}$ K-Krebs solution. However, switchover from such medium to $30 \mathrm{~mm}$ K-Krebs solution (normotonic) almost completely prevented the explosive secretion of $\mathrm{CA}$ otherwise observed after such a switchover in $5.9 \mathrm{~mm}$ K-Krebs solution. This represented over a $90 \%$ inhibition of the explosive secretion by $30 \mathrm{~mm} \mathrm{~K}$ during switchover. To ensure that the inhibitory effect of excess $K$ was a real one, we repeated the identical protocol in the same adrenal gland without increasing $K$ concentration of the medium over $5.9 \mathrm{~mm}$. Figure 9 shows that immediately after the changeover from hypertonic to normotonic solution, the secretion of CA increased over $1700 \mathrm{ng}$ in a $10 \mathrm{~min}$ period.

The antagonism between excess $K$ and changes in tonicity of the perfusion media in producing explosive secretion of $\mathrm{CA}$ was examined on the accumulation of $\mathrm{C}^{14}$-TPP. As can be secn in Figure 8 , the accumulation of $C^{14}-T P P$ of about $190 \mathrm{pg} / \mathrm{mg}$ in 


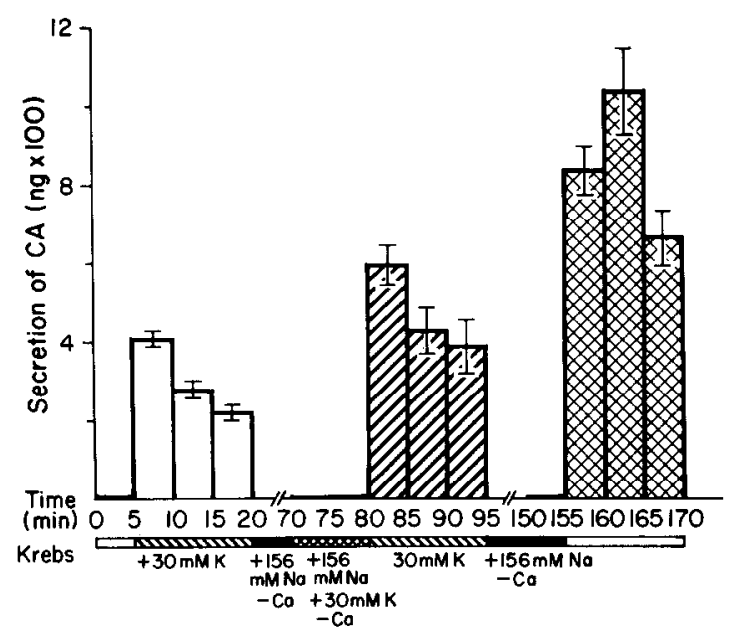

Figure 9. Inhibition by excess $\mathrm{K}$ of explosive secretion of CA evoked by changes in tonicity of the perfusion medium. After $30 \mathrm{~min}$ perfusion with Krebs solution, one $5 \mathrm{~min}$ sample $(0-5 \mathrm{~min})$ was collected to determine spontaneous secretion of $\mathrm{CA}$, and then medium was changed to $30 \mathrm{~mm}$ K-Krebs solution for $15 \mathrm{~min}$. The gland was then perfused with $\mathrm{Ca}$-free and $275 \mathrm{~mm} \mathrm{NaCl}-\mathrm{Krebs}$ solution for $50 \mathrm{~min}$, followed by $30 \mathrm{~mm} \mathrm{~K}$-, Ca-free, and $275 \mathrm{~mm} \mathrm{NaCl}-\mathrm{Krebs}$ solution for $10 \mathrm{~min}$. The medium was changed over to $30 \mathrm{~mm} \mathrm{~K}-\mathrm{Krebs}$ solution and three $5 \mathrm{~min}$ samples were collected. Finally, the medium was changed to $\mathrm{Ca}$-free and $275 \mathrm{~mm} \mathrm{NaCl}-\mathrm{Krebs}$ solution for $60 \mathrm{~min}$, followed by regular Krebs solution. In this series of experiments, $30 \mathrm{~mm} \mathrm{~K}$ was added to the Krebs solution without removing equivalent amounts of $\mathrm{NaCl}$. Each column represents a mean of 4 observations. Vertical lines, SEM.

hypertonic medium was reduced to about $50 \mathrm{pg} / \mathrm{mg}$, if $30 \mathrm{~mm}$ $\mathrm{K}$ was present in the medium. The inhibition of $\mathrm{C}^{14}$-TPP accumulation was associated with a reduction in secretion, just as described in the previous section.

\section{CA content of the adrenal medulla}

Since the secretion of CA was of such an immense magnitude in the present study, it was of interest to determine the CA content of the adrenal gland. The results are shown in Figure 10. Perfusion of the adrenal gland with Krebs solution for over $2 \mathrm{hr}$ did not modify CA content, as compared with that of the unperfused contralateral control gland. However, after perfusion with hypertonic and $\mathrm{Ca}$-free medium for $60 \mathrm{~min}$, if the perfusion was changed over to regular medium for $60 \mathrm{~min}$, there was about a $70 \%$ reduction in CA content. As shown above in several experiments, this treatment caused a large increase in the secretion of CA $(8.25 \pm 0.17 \mu \mathrm{g} ; n=4)$. Figure 10 shows that the reduction in CA content was not associated with any significant loss in the lactate dehydrogenase content of the same adrenal glands.

\section{Discussion}

It was surprising to observe that perfusion of the rat adrenal gland with hypertonic Krebs solution for prolonged periods of time had no adverse effect on the amounts of CA that were secreted spontaneously, or those evoked by excitation of chromaffin cells via splanchnic nerve terminals. Although the secretion of CA was not affected during perfusion with the hypertonic medium, it was increased over 10 -fold immediately upon restoring the perfusion medium to a normal physiological salt solution. The secretory response lasted more than $1 \mathrm{hr}$ after the switchover, and was reproducible in the same adrenal gland.

Certain basic characteristics of this unusual secretory phenomenon were established, such as exposure time and degree of hypertonicity, and the types of agent used to make up the
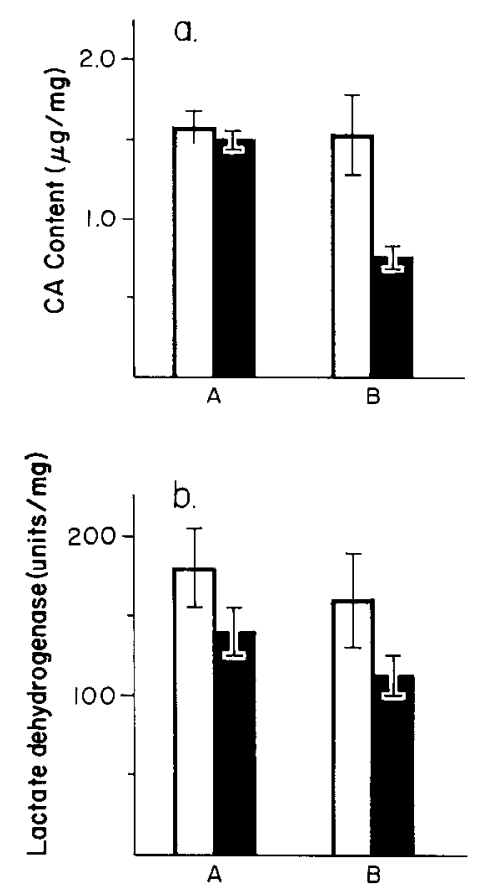

Figure 10. CA and lactate dehydrogenase contents of the adrenal gland after evoking explosive secretion of CA. The left adrenal gland was perfused with Krebs solution for $150 \mathrm{~min}$, and the medulla removed to determine CA content (solid bar in a). In another group, the protocol was identical to that described in Figure 4 (solid bar in b). For both groups, the contralateral right adrenal gland was removed and assayed for CA (open bars). Each medulla was homogenized in ice-cold $0.1 \mathrm{M}$ phosphate buffer $(\mathrm{pH} 7.5)$ and used for estimation of CA $(a)$ and lactate dehydrogenase $(h)$, as described in Materials and Methods. Fach har is mean of 4 experiments. Vertical lines, SEM.

hypertonic Krebs solution. It was essential that the adrenal gland be exposed to hypertonic solution for at least $15 \mathrm{~min}$, and that a sodium chloride concentration be $1.75 \times$ the normal value to initiate the secretion in the postperfusion period with normal medium. Hypertonicity resulting from excess sodium or chloride ions was not the major reason for this unusual secretory phenomenon, because the increase in tonicity achieved by addition of choline chloride, arginine hydrochloride, sodium thiocyanate, and sucrose gave essentially similar results as those achieved with excess sodium chloride.

The primary site from which the secretion begins after changeover from hypertonic to normotonic solution has been determined. Experiments with the antagonists of nicotinic and muscarinic receptors and chronically denervated adrenal glands ruled out the possibility of activation of the cholinergic receptors of chromaffin cells by ACh liberated from splanchnic nerves during switchover from hypertonic to normal Krebs solution. Thus, the effect of the hypertonic medium appears to be directly on the chromaffin cell and not on presynaptic splanchnic nerve terminals.

The importance of $\mathrm{Ca}$ ions in the secretion of CA by a variety of secretagogues has been amply documented (Baker and Knight, 1984; Douglas, 1968; Douglas and Rubin, 1961; Knight and Kesteven, 1983; Viveros, 1975). In the present study we found that $\mathrm{Ca}$ was essential for the secretion of CA evoked by changes in tonicity of the perfusion medium, since the secretory response was virtually absent if $\mathrm{Ca}$ ions were omitted from the normal medium, or if Mn was added to the medium. Most important, the accumulation of $\mathrm{Ca}^{45}$ increased almost 6-fold during switchover from hypertonic to normotonic medium. The need for $\mathrm{Ca}$ suggests that cxocytosis must have becn a major mechanism of 
secretion of CA in the present study as well. Although secretion of other vesicular markers was not monitored, lack of increase in the secretion of the cytoplasmic enzyme, lactate dehydrogenase, eliminated the possibility of leakage of CA via cytoplasm.

The major question is how extracellular $\mathrm{Ca}$ ions are made available to the secretory process when the environment of chromaffin cells is changed from hypertonic to normal. Currently, it is believed that $\mathrm{Ca}$ channels are opened either by activation of nicotine receptors of the chromaffin cell membrane (Kilpatrick et al., 1982; Knight and Kesteven, 1983; Viveros, 1975; Wakade et al., 1986) or by depolarization of the cellular membrane (Hagiwara and Byerly, 1981). The possibility that cholinergic receptors and $\mathrm{Na}$ action potentials are the primary stimulus for opening $\mathrm{Ca}$ channels of chromaffin cells has been eliminated in the present study (Table 2). However, it is likely that electrochemical properties of chromaffin cell membrane may change during perfusion with hypertonic medium. One hour exposure to hypertonic solution may cause a loss of cellular fluid, causing shrinkage of chromaffin cells. As a result, intracellular concentration of $\mathrm{K}$ may increase and lead to hyperpolarization of chromaffin cell membrane, as one would expect from the electrophysiological properties of cell membrane. If indeed the uptake of $\mathrm{C}^{14}$-TPP is inversely related to the membrane potential, as many reports show (see Results), then the more than a 2 -fold increase in $\mathrm{C}^{14}$-TPP accumulation seen in the adrenal medulla perfused with hypertonic solution must reflect a considerable degree of hyperpolarization. And when the perfusion medium was changed from hypertonic to normotonic solution, the accumulation of $\mathrm{C}^{14}$-TPP declined toward the control value. This observation suggests that the hyperpolarized membrane returned toward a more positive membrane potential. Since, during this maneuver, the secretion of CA commences, it is our opinion that the relative change in membrane potential was responsible for opening voltage-sensitive $\mathrm{Ca}$ channels and for an increase in cytostolic levels of Ca ions (see below). In support of such a proposal, studies demonstrated that if the cell membrane was depolarized by prior exposure to $30 \mathrm{~mm} \mathrm{~K}$, then the changes induced by hypcrtonic medium were prevented. The inhibition by excess $\mathrm{K}$ was obtained on the $\mathrm{C}^{14}$-TPP accumulation (Fig. 8), as well as on CA secretion (Fig. 9).

A number of observations rule out the question of tissue damage and lysis in the present phenomenon. It is remarkable that an explosive secretion of CA (from 4 to $4000 \mathrm{ng}$ ) obtained after changes in tonicity of the medium did not result in the release of the cytoplasmic enzyme, lactate dehydrogenase. Furthermore, several interventions reversibly prevented the explosive secretion in a specific manner. For example, when $3 \mathrm{~mm}$ $\mathrm{Mn}$ or $35 \mathrm{~mm} \mathrm{~K}$ were added to the medium prior to switchover from $\mathrm{Ca}$-free hypertonic to normotonic solution, the secretory response was virtually absent, and removal of these agents caused the expected secretion in the same adrenal glands. Most important, the massive secretion - which was associated with about $70 \%$ reduction in CA content of the adrenal medulla-did not produce any alteration in the lactate dehydrogenase content of the tissue.

The massive and prolonged secretory response that begins immediately after switchover from hypertonic to normotonic medium cannot be a result of transient entry of $\mathrm{Ca}$ ions within the first few seconds after voltage-sensitive $\mathrm{Ca}$ channels open, or a gradual disposition of free cytostolic $\mathrm{Ca}$ ions over the next $40 \mathrm{~min}$. It is likely that $\mathrm{Ca}$ channels may remain in an open state for a very long period of time once they are opened by changes in tonicity of the medium. It is clear from the results of Figure 6 that even if $\mathrm{Ca}$ was presented to the adrenal medulla as late as $30-120 \mathrm{~min}$ after the switchover from hypertonic and $\mathrm{Ca}$-free to normotonic solution, $\mathrm{Ca}$ was still transported inside the chromaffin cells and was effective in evoking the secretion of CA.

In summary, an entirely new procedure is introduced to evoke CA secretion in a Ca-dependent manner that should help to explore the properties of Ca channels, storage, and turnover of CA as well as other constituents of chromaffin granules.

\section{References}

Anton, A. H., and D. F. Sayre (1962) A study of the factors affecting the alumina oxide-trihydroxyindole procedure for the analysis of catecholamines. J. Pharmacol. Exp. Ther. 138: 360-375.

Baker, P. F., and D. R. Knight (1984) Calcium control of exocytosis in bovine adrenal medulla cells. Trends Neurosci. 6: 120-126.

Baker, P. F., and T. J. Rink (1975) Catecholamine release from bovine adrenal medulla cells. Trends Neurosci. 6: 120-126.

Douglas, W. W. (1968) Stimulus-secretion coupling: The concept and clues from chromattin and other cells. Br. J. Pharmacol. 34: 451-474.

Douglas, W. W., and R. P. Rubin (1961) The role of calcium in the secretory response of the adrenal medulla to acetylcholine. J. Physiol. (Lond.) 159: 40-57.

Douglas, W. W., and R. P. Rubin (1963) The mechanism of catecholamine release from the adrenal medulla and the role of calcium in stimulus secretion coupling. J. Physiol. (Lond.) 167: 288-310.

Hagiwara, S., and L. Byerly (1981) Calcium channel. Annu. Rev. Neurosci. 4: 69-125.

Kidokoro, Y., A. K. Ritchie, and S. Hagiwara (1979) Effect of tetrodotoxin on adrenaline secretion in the perfused cat adrenal medulla. Nature 278: 63-65.

Kiefer, M., A. J. Blume, and H. R. Kaback (1980) Membrane potential changes during mitogenic stimulation of mouse spleen lymphocytes. Proc. Natl. Acad. Sci. USA 77: 2200-2204.

Kilpatrick, D. L., R. J. Slepetis, J. J. Cocoran, and N. Kirshner (1982) Calcium uptake and catecholamine secretion by cultured bovine adrenal medulla cells. J. Neurochem. 38: 427-435.

Kirpekar, S. M., and P. Cervoni (1963) Effect of cocaine, phenoxybenzamine and phentolamine on catecholamine output from spleen and adrenal medulla. J. Pharmacol. Exp. Ther. 142: 59-70.

Knight, D. E., and N. T. Kesteven (1983) Evoked transient intracellular free $\mathrm{Ca}^{++}$changes and secretion in isolated bovine adrenal medullary cells. Proc. R. Soc. Lond. [Biol.] 218: 177-199.

Lichtshtein, D., H. R. Kaback, and A. J. Blume (1979) Use of lipophilic cation for determination of membrane potential in neuroblastoma-glioma hybrid cell suspension. Proc. Natl. Acad. Sci. USA 76: 630-654.

Marley, E., and W. D. M. Paton (1961) The output of sympathetic amines from the cat's adrenal gland in response to splanchnic nerve activity. J. Physiol. (Lond.) 155: 1-27.

Robinson, R. L. (1967) Stimulation of the catecholamine output of the isolated perfused adrenal gland of the dog by angiotensin and bradykinin. J. Pharmacol. Exp. Ther. 156: 252-257.

Sharma, T. R., and A. R. Wakade (1984) Calcium-dependent secretion of catecholamines following exposure of the rat adrenal gland to hypertonic solution. Pharmacologist 26: 160 .

Viveros, A. H. (1975) Mechanism of secretion of catecholamines from adrenal medulla. In Handbook of Physiology: Endocrinology, Vol. 6, H. Blaschko, A. Sayers, and A. D. Smith, eds., pp. 389-426, American Physiological Society, Washington, DC.

Wakade, A. R. (1981a) Studies on secretion of catecholamines evoked by acetylcholine or transmural stimulation of the rat adrenal gland. J. Physiol. (Lond.) 313: 463-480.

Wakade, A. R. (1981b) Facilitation of secretion of catecholamines from rat and guinea-pig adrenal glands in potassium-free medium or after ouabain. J. Physiol. (Lond.) 313: 418-498.

Wakade, A. R., and T. D. Wakade (1982a) Secretion of catecholamine from adrenal gland by a single electrical shock. Electrotonic depolarization of medullary cell membrane. Proc. Natl. Acad. Sci. USA 79: 3071-3074.

Wakade, A. R., and T. D. Wakade (1982b) Relationship between membrane depolarization, calcium influx and norepinephrine release in sympathetic neurons maintained in culture. J. Pharmacol. Exp. Ther. 223: 125-129.

Wakade, A. R., and T. D. Wakade (1983) Contribution of nicotinic and muscarinic receptors in the secretion of catecholamines evoked 
by endogenous and exogenous acetylcholine. Neuroscience 10:973978.

Wakade, A. R., D. Edgar, and H. Thoenen (1983) Both nerve growth factor and high $\mathrm{K}^{+}$concentrations support the survival of chick embryo sympathetic neurons. Exp. Cell Res. 144: 377-384.

Wakade, T. D., R. K. Malhotra, T. R. Sharma, and A. R. Wakade (1985) Can voltage-sensitive calcium channcls of the rat chromaffin cells be opened at the resting membrane potential? Neuroscience Abstr. 11: 993 .

Wakade, A. R., R. K. Malhotra, and T. D. Wakade (1986) Phorbol ester facilitates $\mathrm{Ca}^{45}$ accumulation and catecholamine secretion by nicotine and excess $\mathrm{K}$ but not by muscarine in the rat adrenal medulla. Nature 321: 698-700.

Wastek, G. J., J. R. Lopez, and E. Richelson (1981) Demonstration of a muscarinic receptor-mediated cyclic GMP-dependent hyperpolarization of the membrane potential of mouse neuroblastoma cells using $\left({ }^{3} \mathrm{H}\right)$ tetraphenylphosphonium. J. Mol. Pharmacol. 19: 15-20. Wilson, S. P., and N. Kirshner (1977) The acetylcholine receptor of the adrenal medulla. J. Neurochem. 28: 687-695.

Wroblewski, F., and J. S. LaDue (1955) Lactic dehydrogenase activity in blood. Proc. Soc. Exp. Biol. Med. 90: 210-216. 